\title{
C. New trends in bioinorganic chemistry
}

\section{C-02}

\section{Myoglobin: from electrostatic fields to gates and tunnels} John S. Olson

Department of Biochemistry and Cell Biology, Rice University, Houston, TX, USA. olson@ rice.edu

Mammalian $\mathrm{Mb}$ has served as a simple model for establishing the basic stereochemical principles that regulate $\mathrm{O}_{2}$ affinity, discrimination between the diatomic gases, and, most recently, the pathways governing the rates of ligand binding. The association equilibrium constant for $\mathrm{O}_{2}$ binding is governed by proximal geometry, which regulates iron reactivity, steric hindrance, which inhibits the binding of all ligands, and hydrogen bonding with polar residues, which preferentially favors $\mathrm{O}_{2}$ binding.

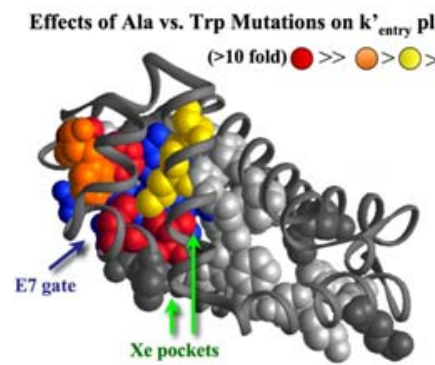

Sperm whale myoglobin for SW Mb and CerHb

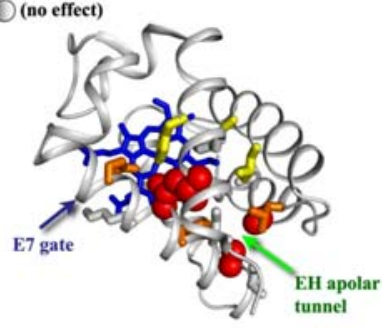

Cerebratulus hemoglobin
Pathways for $\mathrm{O}_{2}$ binding have been mapped by mutagenesis (see figure above), time resolved crystallography, and ultrafast kinetic techniques. In vertebrate myoglobins and hemoglobins, ligands enter and escape by the distal His(E7) gate, whereas in the mini-globin from the sea worm Cerebratulus lacteus an alternative apolar tunnel between the $\mathrm{E}$ and $\mathrm{H}$ helices is used, demonstrating that alternative routes do occur in globins.

\section{C-03}

Searching for ligand migration pathways in myoglobin: a new look into the old problem Alexey Onufriev ${ }^{1}$

${ }^{1}$ Departments of Computer Science and Physics, Virginia Tech, Blacksburg, USA. alexey@cs.vt.edu

No consensus yet exists on the atomic level mechanism by which small non-polar ligands move between surrounding solvent and the binding site buried inside myoglobin. To map out the pathways of ligand migration we use a combination of two approaches:

(1) Analysis of multi-microsecond, room temperature molecular dynamics trajectories in which the $\mathrm{CO}$ ligand shuttles between the internal binding site and the solvent; and (2) pattern and structural origins of transient voids available for ligand migration. Localized transient fluctuations on picosecond time-scale are the primary physical origin of the migration pathways for small non-polar ligands inside the protein. There are two discrete dynamical pathways for ligand migration in myoglobin. The natural propensity of larger fluctuations to occur in the "softer" domains of the protein matrixin-between the helices and in the loop regions-determine the preferential location of the pathways relative to protein scaffold [1].

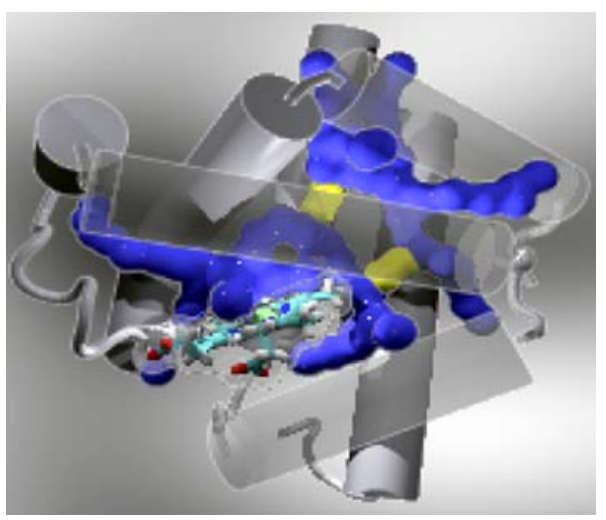

\section{Reference}

1. Ruscio JZ, Kumar D, Shukla M, Michael G. Prisant, T. M. Murali, Onufriev AV (2008) Proc Natl Acad Sci USA 15:9204-9209 (2008).

\section{C-04}

Time-resolved crystallographic studies of heme proteins Vukica Srajer ${ }^{1}$, Reinhard Pahl ${ }^{1}$, Robert Henning ${ }^{1}$, Timothy Graber ${ }^{1}$, Marius Schmidt ${ }^{2}$, James Knapp ${ }^{3}$ and William E. Royer ${ }^{4}$

${ }^{1}$ Center for Advanced Radiation Sources, The University of Chicago, Chicago IL, USA.

${ }^{2}$ University of Wisconsin-Milwaukee, Milwaukee, WI, USA.

${ }^{3}$ Department of Biomedical Science, Mercer University School of Medicine, Savannah, GA, USA.

${ }^{4}$ University of Massachusetts Medical School, Worcester, MA, USA. v-srajer@uchicago.edu

The ultimate goal of time-resolved crystallographic studies of biological macromolecules is to visualize intermediate states along a reaction pathway at atomic resolution, without trapping of the intermediates by chemical or physical methods. This is accomplished by taking X-ray snapshots of the molecule in the crystal as the reaction proceeds at room temperature following the reaction initiation. The technique has reached a mature phase with demonstrated ability to detect small structural changes on ns and sub-ns time scale [1-9] and with important advances in the analysis of time-resolved crystallographic data, such as the use of singular value decomposition (SVD) method to determine the structures of intermediates and elucidate the reaction mechanism [3-4]. We present results of time-resolved crystallographic studies of heme proteins: structural relaxation processes in myoglobin [1] and 
allosteric action in real time in a more complex, cooperative dimeric hemoglobin [8]. These pump-probe time-resolved experiments were conducted at the BioCARS beamline 14-ID at the advanced photon source (USA).

\section{References}

1. Schmidt et al (2005) PNAS 102:11704

2. Ihee et al (2005) PNAS 102:7145

3. Rajagopal et al (2005) Structure 13:55

4. Schmidt et al (2004) PNAS 101:4799

5. Schotte et al (2003) Science 300:1944

6. Bourgeois et al (2006) PNAS 103:4924

7. Šrajer et al (2001) Biochemistry 40:13802

8. Knapp et al (2006) PNAS 103:7649

9. Key et al (2007) Biochemistry 46:4706

\section{C-05}

Probing protein structural dynamics with time-resolved $\mathrm{X}$-ray solution scattering: applications to heme proteins Hyotcherl Ihee

Center for Time-Resolved Diffraction, Department of. Chemistry, KAIST, Daejeon 305-701, Republic of Korea. ihee57@gmail.com The three-dimensional structure of proteins and its evolution in time is essential for understanding protein function and unraveling the structure-function relationship. Currently however, the time resolution of direct structural techniques to track protein conformation changes, such as small-angle X-ray scattering and nuclear magnetic resonance, is limited to at best 0.16 millisecond for solution phase studies. Using short X-ray pulses from a synchrotron, we have developed a pump-probe technique (TR-WAXS) for probing protein structural dynamics in solution with a $10^{6}$-fold improved time resolution of $0.1 \mathrm{~ns}$ that allows for studying tertiary and quaternary structural changes in space and in time. The wide applicability of the TR-WAXS technique is demonstrated for three systems: the tertiary/ quaternary structural transition in human hemoglobin, the tertiary relaxation in myoglobin and the folding of cytochrome c. TR-WAXS is readily used to follow irreversible reactions as illustrated here with the folding of cytochrome $\mathrm{c}$ as well as reversible reactions such as ligand reactions in heme proteins. We expect that TR-WAXS will complement time-resolved X-ray crystallography by probing the motions of proteins in their natural environment.

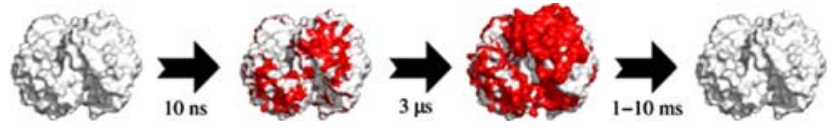

\section{References}

1. Ihee H (2009) Acc Chem Res 42:356-366

2. Cammarata M, Levantino L, Schotte F, Anfinrud PA, Ewald F, Choi J, Cupane A, Wulff M, Ihee H (2008) Nat Methods 5:881-887

\section{C-06}

Resonant X-ray absorption by ${ }^{57} \mathrm{Fe}$ selectively probes structure and energetics of heme proteins

\section{J. Timothy Sage}

Department of Physics and Center for Interdisciplinary Research on Complex Systems, Northeastern University, Boston, MA, USA.

jtsage@neu.edu

Vibrational motions probe molecular structure and energetics on picosecond and subpicosecond time scales. Nuclear resonance vibrational spectroscopy (NRVS) determines the complete vibrational density of states (VDOS) of ${ }^{57} \mathrm{Fe}$ at protein active sites. Myoglobin provides an ideal illustration of the unique information revealed by this novel method, because of the easy availability of high ${ }^{57} \mathrm{Fe}$ concentrations and because of its utility for modelling more complex heme proteins. All Fe-ligand vibrations contribute to the NRVS signal, providing a highly selective probe for local structure. In one recent example, we compared NRVS observations with quantum chemical vibrational predictions to establish unambiguous vibrational signatures for protonation of monooxygenated hemes, and conclusively demonstrated that the reactive compound II intermediate is unprotonated. The VDOS yields interpretable information on molecular structure and energetics, even in advance of the traditional intermediate step of resolving and identifying individual vibrational modes whose frequencies display structural sensitivity. Fe-ligand vibrations make the primary contribution to the stiffness, an effective force constant which measures the average strength of nearest neighbor interactions, while low frequency oscillations of the protein determine the resilience, a distinct force constant that probes the elasticity of the heme environment.

\section{C-07}

Crystallographic refinement of metal redox states in metalloproteins

Douglas C. Rees ${ }^{1}$, Holger Dobbek ${ }^{2}$, Jacques Meyer ${ }^{3}$, Susana Andrade ${ }^{4}$, Oliver Einsle ${ }^{4}$

${ }^{1}$ Division of Chemistry 114-96, HHMI, California Institute of Technology, Pasadena, CA 91125 USA.

${ }^{2}$ Laboratorium Proteinkristallographie, Universität Bayreuth, 95440 Bayreuth, Germany.

${ }^{3}$ Biophysique Moléculaire et Cellulaire, DRDC, CEA-Grenoble, 38054 Grenoble, France.

${ }^{4}$ Albert-Ludwigs-University Freiburg, Institute for Organic Chemistry and Biochemistry, 79104 Freiburg, Germany. dcrees@caltech.edu While the position of an X-ray absorption edge depends primarily on the element and coordination environment, it is also sensitive to oxidation state; for example, the edge positions between ferrous to ferric iron can shift by +1 to $+5 \mathrm{eV}$. Due to these absorption effects, the atomic scattering factor for X-rays will include terms that correspond to the wavelength dependent real (dispersive) and imaginary (absorption) corrections to the scattering factor. Following the analysis of $\mathrm{Wu}$ et al. [1] to identify the oxidation states of iron in a small molecule complex, we have developed a method [2] to derive anomalous scattering contributions for individual atoms within a protein crystal by collecting diffraction data at multiple energies across the relevant X-ray absorption edge. The method has been applied to a [2Fe:2S] ferredoxin system with localized charges in the reduced state of the iron-sulfur cluster. An important goal is to apply this approach to the nitrogenase molybdenum iron-protein to assess the oxidation states for each of the 17 distinct iron sites in this structure. Our progress towards this objective will be described, particularly emphasizing the relative oxidation states of the irons in the FeMo-cofactor and the P-cluster of the MoFe-protein.

\section{References}

1. Wu G, Zhang Y, Ribaud L, Coppens P, Wilson C, Iversen BB, Larsen FK (1998) Inorg Chem 37:6078-6083

2. Einsle O, Andrade SLA, Dobbek H, Meyers J, Rees DC (2007) J Am Chem Soc 129:2210-2211 


\section{C-08}

Metal speciation for metalloproteomics using X-ray absorption spectroscopy

Robert A. Scott ${ }^{1,2}$, Uma Mahendra Kumar Koppolu', Michael W. W. Adams' ${ }^{2}$, Angeli L. Menon², Aleksandar Cvetkovic ${ }^{2}$, Joseph Scott ${ }^{2}$, Britt Hedman ${ }^{3}$, Matthew J. Latimer ${ }^{3}$

${ }^{1}$ Department of Chemistry, University of Georgia, Athens, GA 30602 USA.

${ }^{2}$ Department of Biochemistry and Molecular Biology, University of Georgia, Athens, GA 30602 USA.

${ }^{3}$ Stanford Synchrotron Radiation Lightsource, SLAC National Accelerator Laboratory, Menlo Park, CA 94025 USA.

rscott@uga.edu

High-throughput X-ray absorption spectroscopy (HT XAS) is being developed as an adjunct structural proteomics technology for investigating the "metalloproteome", all metalloproteins in the proteome of an organism at a given time, grown under a given condition. Metalloproteomics will provide global information about the cellular response to environmental signals focused on the expression, loading, and assembly of metalloproteins. In creating a pipeline from genome to metalloproteome, several bottlenecks to high-throughput determination of metal-site structure must be overcome. For example, automation of arraying small samples for XAS examination must be invented, automation of rapid data collection of multiple low-volume, low-concentration samples must be developed, automation of data reduction and analysis must be perfected. HT XAS requires the separation of intact (native) proteome components. It is likely that solution of this initial proteomic "separation technology" problem will be the key to removing a major roadblock to the creation of this technology. Feasibility studies to demonstrate this technology will be presented and initial experiments based on native proteome fractionation discussed. The use of this technology to investigate metal-dependent expression and assembly of the metalloproteome under stress of essential metal depletion or toxic heavy metal exposure is planned.

\section{C-09}

\section{Transient interactions between redox proteins}

\section{Marcellus Ubbink ${ }^{1}$}

${ }^{1}$ Inst. Chemistry, Leiden University, Gorlaeus Laboratories, Einsteinweg 55, 2333 CC Leiden, The Netherlands.

m.ubbink@chem.leidenuniv.nl

Protein complexes formed as part of electron transfer chains must be short-lived in order not to limit the electron flow. Electron transfer proteins react with more than one partner, usually using a single interface via a ping-pong mechanism.

These transient complexes can be visualized in atomic detail using nuclear magnetic resonance (NMR) spectroscopy. With paramagnetic tags for NMR the degree and amplitude of the dynamics in these complexes can be established. It is demonstrated that for a large fraction of the time the complex exists in a dynamic, nonspecific encounter ensemble, in which the partners role over each other. These complexes balance on the brink of non-specificity. These findings will be illustrated with the electron flow from methylamine to oxygen in Paracoccus denitrificans. The whole chain,

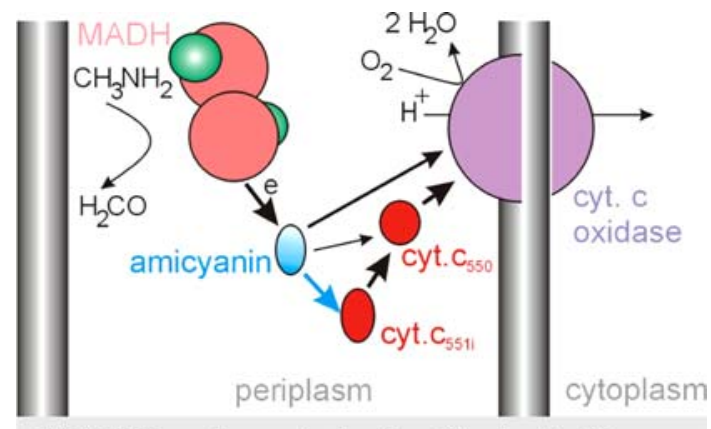

MADH redox chain in $P$. denitrificans. The arrow line widths indicate the speed of ET. Blue line means up-hill reaction.

comprising methylamine dehydrogenase (MADH), amicyanin, several cytochromes $c$ and cytochrome $c$ oxidase could be reconstituted in vitro and the flow of electrons followed. In parallel, the complexes were characterized by NMR. The results suggest that such peripheral routes that allow bacteria to metabolize a variety of substrates, work in a 'plug-and-grow' mode, without a need for highly specific electron acceptors, even involving up-hill electron transfer steps.

\section{C-10}

Structural mapping and interfacial electron transfer of redox metalloproteins at the single-molecule level Jingdong Zhang, Qijin Chi, Palle S. Jensen, Allan G. Hansen, Anna C. Welinder, Jens Ulstrup

DTU Chemistry and Nano DTU, Building 207, Technical University of Denmark, DK-2800 Kgs. Lyngby, Denmark. ju@kemi.dtu.dk

Voltammetry of molecular monolayers of redox metalloproteins offers comprehensive mechanistic detail of surface-confined protein molecules. Limitations are ill-defined microenvironments at the polycrystalline electrode surfaces nearly always employed. Singlecrystal, atomically planar electrode surfaces have opened routes to improved voltammetric resolution and to the introduction of scanning probe microscopies, particularly scanning tunnelling microscopy directly in aqueous biological media (in situ STM). In situ STM offers structural mapping and has disclosed even new electron transfer (ET) phenomena at the single-molecule level. Electronic conductivity of single molecules in aqueous media is described in terms of superexchange, electron hopping, and coherent multi-electron transfer. Transfer to aqueous media imposes drastic differences in the ET mechanisms, caused by strong solvent effects, reflected in intriguing current-overpotential and current-bias voltage relations $[1,2]$.

We overview first some cases of condensed matter single-molecular conductivity of transition metal complexes, organic redox molecules and molecular-scale metallic nanoparticles for which details of the conduction mechanism have been reported. We show next that these views extend both to small redox metalloproteins and to more complex metallo enzymes. 


\section{References}

1. Hammerich O, Ulstrup J (eds) (2008) Bioinorganic electrochemistry. Springer, Dordrecht

2. Zhang J, Kuznetsov AM, Medvedev IG, Chi Q, Albrecht T, Jensen PS, Ulstrup J (2008) Chem Rev 108:2737-2791

\section{C-11}

Ultrafast 2D-IR vibrational echo spectroscopy of heme proteins

Haruto Ishikawa $^{1,2}$, Michael D. Fayer ${ }^{2}$

${ }^{1}$ Department of Chemistry, Grad. Sch. Sci. Osaka University, Toyonaka 560-0043, Japan.

${ }^{2}$ Department of Chemistry, Stanford University, CA 94305 USA. haruto@chem.sci.osaka-u.ac.jp

We have investigated protein structural dynamics on short time scales (100 fs to $100 \mathrm{ps}$ ) using ultrafast two dimensional (2D-IR) vibrational echo spectroscopy. Under thermal equilibrium conditions, a protein's structure is constantly fluctuating among conformations associated with different positions on the broad rough minimum on the free energy landscape. Although different conformational substates may be apparent in a vibrational absorption spectrum, linear IR absorption spectra cannot provide information on structural dynamics because dynamical information is masked by inhomogeneous broadening of the lineshapes. 2D-IR vibrational echo spectroscopy makes structural fluctuations a direct experimental observable.

The conformational dynamics are directly measured through the growth of cross-positions peaks in the 2D-IR spectra. In the case of heme proteins, $2 \mathrm{D}$-IR measurements utilize heme ligated $\mathrm{CO}$ vibration as a direct sensor of a protein's dynamics measured at the active site. Since different conformers of a protein undergo characteristic motions, which lead to distinct time-dependent spectral changes, 2DIR vibrational echo experiments can directly characterize. Conformational switching between two well-defined substates of a myoglobin mutant is observed on $\sim 50 \mathrm{ps}$ timescale. The conformational switching results in a change in the location of the side group of the distal histidine which is detected by the time dependence of the 2D-IR spectra. These results demonstrate that protein can interconvert between conformational substates on time scales much faster than has been appreciated previously. We have also succeeded in estimating the rates for structural fluctuations of neuroglobin, horse radish peroxidase, and cytochrome $c$.

\section{C-12}

Development of a generic approach to native metalloproteomics: application to the quantitative identification of soluble copper proteins is Escherichia coli and tungsten proteins in Pyrococcus furiosus Sevcenco AM ${ }^{1,2}$, Krijger GC ${ }^{2}$, Pinkse MW ${ }^{1}$, Verhaert PDEM ${ }^{1}$, Hagen $W^{1}{ }^{1}$, Hagedoorn PL ${ }^{1}$

${ }^{1}$ Department of Biotechnology, Delft University of Technology, Julianalaan 67, 2628 BC, Delft, The Netherlands.

${ }^{2}$ Department of Radiation, Radionuclides and Reactors, Delft Univeristy of Technology, Mekelweg 15. p.1.hagedoorn@tudelft.nl A combination of techniques to separate and quantify the native proteins associated with a particular transition metal ion from a cellular system has been developed [1]. The procedure includes four steps: (1) labeling of the target proteins with a suitable short-lived radioisotope (suitable isotopes are ${ }^{64} \mathrm{Cu},{ }^{67} \mathrm{Cu},{ }^{99} \mathrm{Mo},{ }^{69} \mathrm{Zn},{ }^{56} \mathrm{Mn}$,
${ }^{65} \mathrm{Ni}$ ); (2) separation of intact soluble holoproteins using native isoelectric focusing combined with blue native polyacrylamide gel electrophoresis into native-native 2D gel electrophoresis; (3) spot visualization and quantification using autoradiography; and (4) protein identification with tandem mass spectrometry. The method was applied to the identification of copper protein from a soluble protein extract of wild-type Escherichia coli K12 using the radioisotope ${ }^{64} \mathrm{Cu}$. The $E$. coli protein $\mathrm{CueO}$, which has previously been only identified as a multicopper oxidase following homologous overexpression, was now directly detected as a copper protein against a wild-type background at an expression level of $0.007 \%$ of total soluble protein. The finding that the periplasmic protein $\mathrm{CueO}$ is the only major form of soluble protein bound copper in E. coli strengthens the view that the bacterial periplasm contains only a few periplasmic copper proteins, and that the cytosol is devoid of copper proteins. Investigation of the tungsten proteome of Pyrococcus furiosus is in progress.

\section{Reference}

1. Sevcenco AM, Krijger GC, Pinkse MW, Verhaert PDEM, Hagen WR, Hagedoorn PL (2009) J Biol Inorg Chem (in press)

\section{C-13}

\section{The reaction mechanism of multi-copper oxidases} studied by theoretical methods

Ulf Ryde ${ }^{1}$, Lubomir Rulíšek ${ }^{2}$

${ }^{1}$ Deptartment of Theoretical Chemistry, Lund University, P. O. Box 124, 22100 Lund, Sweden.

${ }^{2}$ Gilead Sciences and IOCB Research Center, Institute of Organic Chemistry and Biochemistry, Academy of Sciences of the Czech Republic, Flemingovo nám. 2, 16610 Praha 6, Czech Republic. Ulf.Ryde@teokem.lu.se

The multi-copper oxidases catalyse the four-electron reduction of $\mathrm{O}_{2}$ to water, coupled to the oxidation of various substrates. The former reaction takes place in a trinuclear copper cluster that is linked to a mononuclear blue-copper site. We have studied several aspects of this reaction using theoretical methods.

- We have identified the structures of key intermediates in the reaction, especially the peroxy and native intermediates by QM/ MM methods [1].

- We have used multiconfigurational methods to calculate spectroscopic properties of these intermediates [2].

- We have combined EXAFS refinements with QM/MM calculations to get a direct estimate of what structures fit experimental data [3].

- We have estimated the activation energies involved in the reduction of $\mathrm{O}_{2}$ [1].

- We have calculated reduction potentials for the various intermediates in the reaction cycle.

- We have estimated reorganisation energies for the two copper sites.

Several of these results will be discussed in this talk.

\section{References}

1. Rulîšek L, Solomon EI, Ryde U (2005) Inorg Chem 44:5612

2. Chalupský J, Neese F, Solomon EI, Ryde U, Rulíšek L (2006) Inorg Chem 45:11051

3. Ryde U, Hsiao Y-W, Rulíšek L, Solomon EI (2007) J Am Chem Soc 129:726-727 